\section{Noncategorical perception of a voiced stop: A replication $* \dagger$}

\author{
J. RICHARD BARCLAY †† \\ University of Minnesota, Minneapolis, Minnesota 55455
}

Several studies have purported to show that perception to stop consonants is categorical, i.e., the stimuli are discriminated only slightly better than they are identified. However, all of these studies have employed successive rather than simultaneous discrimination tasks, confounding the effects of memory with those of immediate perception. The present experiment minimized the role of memory by requiring Ss to identify the same set of synthetic voiced stops twice, first using three response categories $(b, d, g)$ and then using two $(b, g)$. Results indicated that six out of seven $\mathrm{Ss}$ who appeared to be categorical perceivers on the basis of preliminary traditional measures later demonstrated noncategorical perception of $/ d /$. A view of speech processing was suggested whereby consonants are perceived continuously but remembered categorically in terms of an articulatory code.

Speech stimuli which are difficult to discriminate unless they are identified differently (i.e., assigned different phonemic labels) are said to be perceived categorically. Evidence suggesting that consonants, but not vowels, are perceived categorically (Fry, Abramson, Eimas, \& Liberman, 1962; Liberman, Harris, Hoffman, \& Griffith, 1957; Liberman, Harris, Kinney, \& Lane, 1961; Stevens, Ohman, \& Liberman, 1963; Studdert-Kennedy, Liberman, Harris, \& Cooper, 1970) has been used to support the motor theory of speech perception described by Liberman, Cooper, Shankweiler, \& Studdert-Kennedy (1967). However, a recent study by the present $E$ (Barclay, 1970) showed that at least one of the voiced stops $(/ \mathrm{d} /)$ could be perceived noncategorically under appropriate conditions. Thus, previous demonstrations of categorical perception may have inadvertently underestimated listeners' ability to detect intraphonemic variation. The present experiment examined the reliability of the noncategorical perception finding by attempting to replicate it under conditions less favorable to its reappearance than those previously employed.

Demonstrations of categorical

*Preparation of this paper was supported in part by grants to the Univeraity of Minnesota, Center for Regearch in Human Leaming, from the National Sclence Foundation (GB-17690), the National Institute of Child Health and Human Development (HD-00088), and the Graduate School of the University of Minnesota: and to the University of Minnesota, Institute of Child Development. from the National Institute of Mental Health (ST01MH06668-13).

tThe author is grateful to James $J$. Jenkins, Winnitred Strange, and John I. Yellott, Jr., for their helpful advice and criticism.

+ Now at the University of Colorado. perception have commonly used the following procedure. Synthetic speech stimuli varying in equal steps along a chosen acoustic continuum are presented either singly or in sequential triads. Each triad contains two identical stimuli plus a third stimulus one or more steps away. Ss perform two tasks: identification (assigning phonemic labels to the singly presented stimuli) and discrimination, which involves either an ABX or an oddity task. Both discrimination tasks are forced-choice procedures: in the ABX task, the S decides whether the third stimulus of each triad was identical to the first or the second, while in the oddity task he decides which of the three stimuli was different from the other two. Discrimination functions have been accurately predicted from identification functions by making two assumptions: (1) that the $\mathrm{S}$ covertly identifies each stimulus of the triad exactly as he overtly identifies it when presented singly, and (2) that stimuli are discriminable only when identified differently. Thus, at a given degree of acoustic separation, two stimuli identified as the same phoneme are much more difficult to discriminate than two stimuli identified as different phonemes. This agreement between discrimination and identification forms the strongest empirical basis for categorical perception assertions (Studdert-Kennedy et al, 1970).

Note that this use of the ABX and oddity techniques measures only successive discrimination. Thus, within a given triad, the listener must compare what he hears with what he remembers having heard a short time before. It follows that a listener's capacity for discrimination will be impaired to the extent that perceived acoustic information is lost or disorted during the interstimulus interval. If simultaneous discrimination of speech stimuli, unlike successive discrimination, is independent of identification, then the claim that consonants are categorically perceived would be unacceptable. Unfortunately, simultaneous discrimination of speech stimuli is exceedingly difficult to measure directly, since dichotic presentation is subject to complicating laterality and fusion effects (e.g., Halwes, 1969).

In the previous study, this investigator partially circumvented the successive discrimination problem by adding a second identification task to the traditional design described above. The stimuli were synthetic two-formant approximations to the syllable/consonant $+a /$. Second formant transitions varied in equal steps through a range producing, in order of occurrence along the continuum, the voiced stops $/ \mathrm{b} /, / \mathrm{d} /$, and $/ g /$ as initial consonants. In combination, an oddity task and a three-alternative (b, $d$, or $g$ ) identification task (3-AIT) yielded two categorical perceivers according to the traditional criteria. Interpolated between these two tasks was a two-alternative identification task (2-AIT), in which Ss decided whether each singly presented stimulus sounded more like /ba/ or /ga/. Since the same stimuli were used on both identification tasks, during the 2-AIT, a categorical perceiver would have heard several indistinguishable initial $/ d / s$. These perceptually identical sounds would all have sounded either more like $/ \mathrm{b} /$ than like $/ \mathrm{g} /$, or the reverse, or they would all have sounded equally unlike /b/ or $/ g /$. A noncategorical perceiver would not have treated all $/ \mathrm{d} / \mathrm{s}$ as identical sounds, but rather would have parsed the set of $/ d / s$ into those perceptually closer to $/ \mathrm{b} /$ and those perceptually closer to $/ \mathrm{g} /$.

Of the two supposedly categorical perceivers tested on the 2-AIT, one consistently and correctly assigned her /d/s to their respective sides of the continuum. The other $S$, although less accurate, also convincingly evidenced intraphonemic discrimination. Obviously, if simultaneous discrimination were measured directly, it would be at least as fine as that which the 2-AIT measured indirectly by minimizing memory effects. Since these findings contradict conclusions drawn from several other investigations of the perception of voiced stops, an attempt at replication was considered advisable. The present experiment differed from that just described in three ways. First, to reduce boredom and its consequent lapses of attention, fewer trials were 


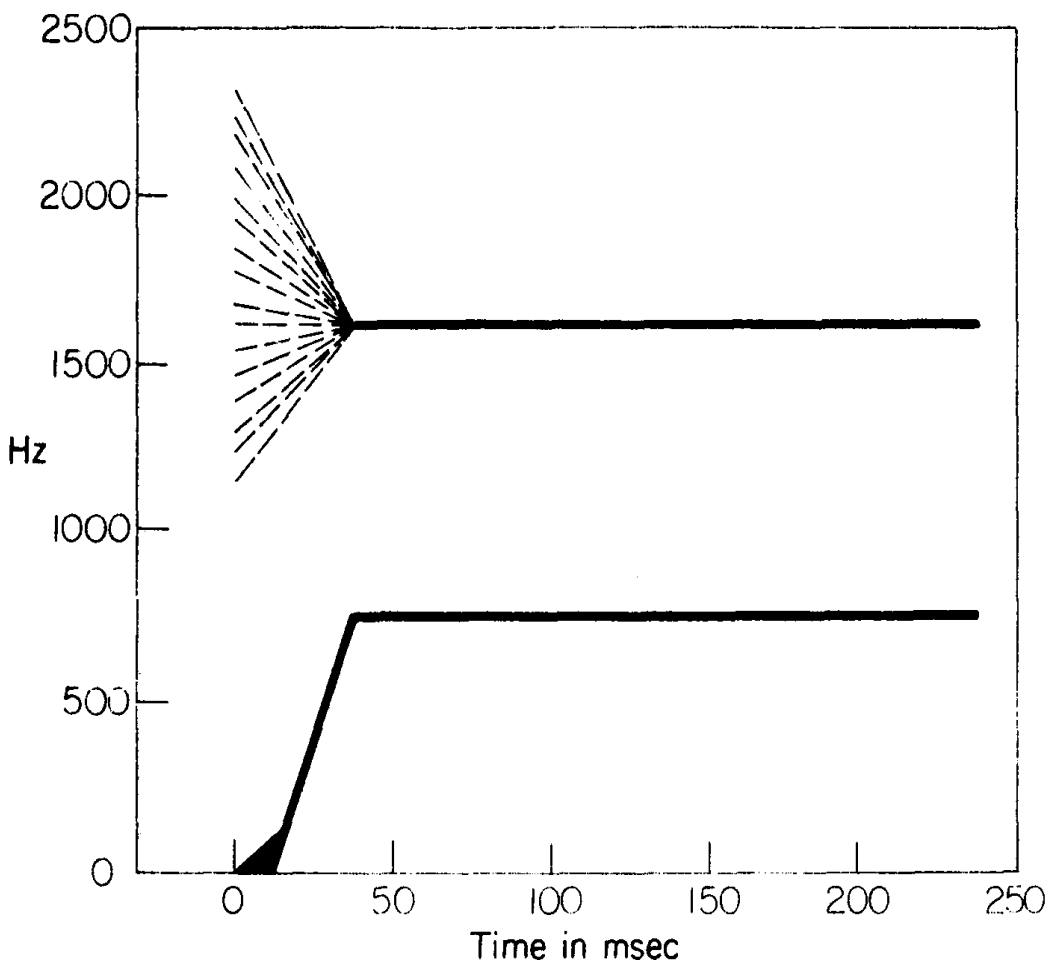

Fig. 1. Schematic representation of the stimuli. Each dotted line represents one of the F2 transitions used.

run on each task. Second, data analysis was modified to better assess the validity of the categorical perception hypothesis. Finally, task order was rearranged: the 2-AIT was presented after the 3-AIT. It was assumed that the experience of overtly identifying $/ \mathrm{d} \approx / \mathrm{s}$ in the 3-AIT would, if anything, interfere with Ss' ability to reclassify them later in the 2-AIT.

\section{METHOD}

Subjects

Eight University of Minnesota female undergraduates volunteered to serve as paid So. One $S$ was subsequently excluded because her unusually inconsistent data were irrelevant to crucial intertask comparisons. Only one of the remaining seven Ss had previously heard synthetic speech, and her data were not systematically different from the rest.

\section{Materials and Apparatus}

The stimuli were two-formant synthetic speech sounds varying only in the direction and extent of the F2 (second formant) transition, an acoustic cue which distinguishes among the voiced stops (Liberman, Delattre, Cooper, \& Gerstman, 1954). Fundamental frequency was held constant at $90 \mathrm{~Hz}$. Following a 15-msec low-amplitude F1 (first formant) at $150 \mathrm{~Hz}$, which represented closure voicing, formants moved comfortable listening level through Koss Pro-600A earphones. The earphones were rotated among Ss across experimental sessions.

\section{Procedure}

The Ss were tested in groups of four on 3 consecutive days. The first session began with a brief familiarization period in which $S$ s listened to synthetically produced words, prose, etc. They were urged and frequently reminded to try to regard these samples and subsequent stimuli as real speech. The demonstration tape was followed by the oddity task, during which each of the two discrimination tapes was presented once. Ss were instructed to decide (forced-choice) which of the three sounds on each trial sounded in any way different from the other two. Before beginning the test trials, Ss listened without responding to 10 triads drawn haphazardly from the middle of the first tape. Response sheets contained three boxes for each trial, corresponding to the first, second, and third stimuli presented. Ss were told to rate their confidence in each response by marking the box of the "odd" stimulus either ++ (very sure), + (fairly sure), or 0 (guessing). In all, each of the 14 different stimulus combinations was presented 12 times for a total of 168 trials.

The second session was devoted to the 3-AIT. Ss understood that the same speech sounds used in the discrimination task would be presented again, this time singly, and that all sounds were syllables ending identically in /æ/ but differing in thein initial consonants. Ss were told to identify each stimulus by writing $b, d$. or $g$ for its initial consonant. Again, 10 examples preceded the actual testing. Testing included two complete presentations of the identification tape (320 trials, a total of 20 presentations per stimulus).

The third session, which focused on the 2-AIT, began with a briel discussion of the major purpose of the experiment. Ss were informed that during the 3-AIT, they had actually assigned each consonant label to a range of physically different sounds. An elementary description of the construction of the stimuli explained that the /d/ range was located between the $/ \mathrm{b} /$ and $\mid \mathrm{g} /$ ranges along the continuum. Thus, E explained, one-half of the /d/ range is adjacent to the $/ \mathrm{b} /$ range and the other half is adjacent to the $/ \mathrm{g} /$ range.

Instructions for the 2-AIT were then delivered: "We want to find out if you can tell which of the sounds you normally call $d$ are near the $b s$, and which are near the gs, in the way we just discussed. I'm going to play 
the same tape you heard yesterday, and this time I want you to decide whether each stimulus sounds more like /ba/ or more like /ga/. Most stimuli will sound obviously like either /bae/ or /gae/, but some will sound most like $/ \mathrm{d} æ /$. If you listen very carefully, you can hear that many of the /dæ/s actually start off like either /ba/ or /ga/ but then change very quickly into /dæ/; that first fraction of a second will give you the information you need for a correct response if you are paying attention. On each trial, just write $b$ or $g$ to indicate whether the stimulus was closer to /ba/ or to /gee/."

The Ss who assign all their /dae/s to only one of the two available response categories might be accurately reporting their perceptions, or they might simply be using the strategy which makes their job as easy as possible. For this reason, Ss were also urged not to lazily assign all /da/s to one category without listening closely to each stimulus. Ten examples and 320 test trials were presented, using the same identification tape presented in the 3-AIT.

\section{RESULTS AND DISCUSSION}

Oddity Task and 3-AIT

The first analysis examined the relationship between successive discrimination and three-category identification. As expected, discrimination functions tended to be marked by peaks between and troughs within the phoneme categories defined by identification functions. Weighting a S's discrimination data by her relative confidence in each response usually exaggerated these discontinuities. The data were weighted according to the method described by Strange and Halwes (1971), which permits one to estimate discrimination functions that would be obtained from many trials by appropriately weighting the responses from the first few. At the outset of the present study, estimated discrimination functions were expected to be useful in various data analyses. However, wide individual differences in Ss' use of the rating scale required that only their raw scores be used, and the weighted functions were not further treated.

The degree of correspondence between discrimination and 3-AIT functions was assessed by statistically comparing obtained discrimination curves with those predicted from identification data. For this purpose, the predictive formulae used by Liberman et al (1957) on ABX data were modified to suit the oddity task. Predictive accuracy rested upon the validity of two assumptions: (1) that the stimuli were perceived categorically, and (2) that Ss used the
Table 1

Goodness of Fit Test on Prodicted vs Obtained Discrimination Scores

\begin{tabular}{lcc}
\hline S & $\chi^{2}(14)$ & $p$ \\
\hline SM & 19.78 & $<.25$ \\
JA & 11.66 & $<.75$ \\
BD & 28.02 & $<.025$ \\
SF & 16.06 & $<.50$ \\
DD & 22.22 & $<.10$ \\
SL & 12.08 & $<.75$ \\
KB & 36.30 & $<.001$ \\
\hline
\end{tabular}

same categories in both oddity and identification tasks. Note that successful prediction would not necessarily imply that the assumption are valid: categorical perception is sufficient but not necessary to produce agreement between identification and successive discrimination, since the latter involves memory as well as immediate perception. This point has apparently been overlooked by those classifying Ss as categorical perceivers according to traditional criteria.

For each $S$, the extent of agreement between predicted and obtained discrimination scores was assessed by a chi-square test of goodness of fit. A fit was judged acceptable if it yielded $a$ nonsignificant $(p>.05)$ chi-square value. The results of the tests are presented in Table 1.

Acceptable fits were found for five of the seven Se. Inspection of the data revealed that for each of the two remaining Ss, the fit would have been acceptable but for a large discrepancy between predicted and obtained scores for a single stimulus. For S K.B., the data for one stimulus accounted for $36 \%$ of the significant chi-square value, and for S B.D., the data for one stimulus (a different one) accounted for $57 \%$ of the significant chi-square value. Thus, the results from this portion of the experiment in isolation support the categorical perception hypothesis and indicate the suitability of the stimuli and apparatus for the tasks involved.

\section{2-AIT}

For each $\mathbf{S}$, certain stimuli were heard as /da/ on some nonzero proportion of their presentations in the 3-AIT; call each such stimulus $\Delta_{i}$. When $\mathbf{S}$ can only label each stimulus $B$ or $G$ in the 2-AIT, the following response probabilities can be computed:

$\mathrm{p}\left(\mathrm{B} \mid \Delta_{\mathrm{i}}\right)=\mathrm{P}\left(\mathrm{b} \mid \Delta_{\mathrm{i}}\right)+\mathrm{P}\left(\mathrm{d} \mid \Delta_{\mathrm{i}}\right) \cdot \theta$,

and

$P\left(G \mid \Delta_{i}\right)=P\left(g \mid \Delta_{i}\right)+P\left(d \mid \Delta_{i}\right)(1-\theta)$, where $B$ and $G$ are labels assigned in the 2-AIT, $b, d$, and $g$ are labels assigned in the 3-AIT, and $\theta$ refers to a bias for responding $B$ to a stimulus which sounded most like /da/. Assuming that the probabilities of hearing any given stimulus as /ba/ and /gæe/ do not diminish from one task to the next, each $P\left(B \mid \Delta_{i}\right)$ and $P\left(G \mid \Delta_{i}\right)$ will correspond exactly to the obtained 2-AIT data, with a $\theta$ value somewhere between 0.00 and 1.00 .

For example, imagine that on the 3-AIT a hypothetical $S$ heard a given stimulus as $/ \mathrm{ba} / 30 \%$ of the time and as /da/ $70 \%$ of the time. During the 2-AIT, we predict that he will again hear it as /bæ/ $30 \%$ of the time and that he will also label it $B$ on some proportion $(\theta)$ of the times when it sounds most like /da/. If his $\theta$ value on that stimulus was 1.00 , he would always label it $B$. If, on the other hand, his $\theta$ value was 0.50 , he would give only $0.30+(0.70)(0.50)=65 \% \mathrm{~B}$ responses; the remaining $35 \%$ of the responses would, of course, be Gs.

If the $S$ is a categorical perceiver, the various $\Delta_{i} s$ are indistinguishable; that is, each / $d /$ he hears is identical to any other /d/ regardless of either the particular stimulus presented or the particular probability of hearing that stimulus as /da/. Thus if perception of the stop consonants is categorical, one should be able to predict 2-AIT performance accurately from 3-AIT data with $\theta$ held constant over the $\Delta_{i} s$ for any given $S$. For instance, $S$ might hear $/ \mathrm{d} /$ as closer to $/ \mathrm{b} /$ than to $/ \mathrm{g} /$ $(\theta=1.00)$, or the reverse $(\theta=0.00)$. If he hears /d/ as equally unlike both alternatives, he might use a random strategy $(\theta=0.50)$ or a probabilistic one. If, however, perception of stop consonants is not categorical, $\theta$ values should change significantly across the $\Delta_{\mathrm{i}} \mathbf{S}$.

For each S, a family of predicted curves was generated in accordance with the categorical perception hypothesis. Each such curve resulted from setting $\theta$ at a fixed value and then solving Eq. 1 for each $\Delta_{i}$ stimulus, with the $\theta$ value varying systematically from curve to curve. A chi-square test of goodness of fit was then used to evaluate the agreement between the obtained data and each predicted curve. If the best fit that could be achieved with a fixed $\theta$ value is still unacceptable (i.e., if it yields a significant chi-square), then one may conclude that the data cannot be predicted accurately from the categorical perception hypothesis. In that case, one could achieve an acceptable fit only by adjusting $\theta$ for each $\Delta_{i}$.

The goodness-of-fit test for each $S$ was performed as follows. For each $\Delta_{i}$, the following chi-square was computed: 
Table 2

Goodness of Fit Test on Predicted vs Obtained 2-AIT Functions

\begin{tabular}{lcccc}
\hline S & Minimal $\chi^{2}(J-1)$ & $\begin{array}{c}\text { Correspond- } \\
\text { ing } \theta\end{array}$ & df & p \\
\hline SM & 9.06 & .42 & 3 & $<.05$ \\
JA & 11.48 & .33 & 2 & $<.005$ \\
BD & 2.61 & .04 & 7 & $<.95$ \\
SF & 8.04 & -.20 & 3 & $<.05$ \\
DD & 26.14 & .39 & 11 & $<.01$ \\
SL & $\mathbf{1 8 . 4 0}$ & .65 & 7 & $<.025$ \\
KB & 30.34 & .58 & 7 & 001 \\
\hline
\end{tabular}

$$
\begin{aligned}
\chi^{2} \Delta_{i}^{\prime}= & \frac{\left[N P *\left(B \mid \Delta_{i}\right)-N P\left(B \mid \Delta_{i}\right)\right]^{2}}{N P\left(B \mid \Delta_{i}\right)} \\
& +\frac{\left[N P *\left(G \mid \Delta_{i}\right)-N P\left(G \mid \Delta_{i}\right)\right]^{2}}{N P\left(G \mid \Delta_{i}\right)}
\end{aligned}
$$

where $\mathrm{N}=20, \mathrm{P} *$ denotes an obtained proportion, $P$ denotes a predicted proportion, and the remaining expressions are as described in Eqs. 1 and 2. In calculating the expected frequencies, $\theta$ was varied (by computer) from 0.00 to 1.00 in steps of 0.01 . Next, the $x^{2} \Delta \mathrm{i}$ s were summed over the $\Delta_{i} \mathrm{~s}$ at each level of $\theta$, yielding at each level a chi square with $J-1$ degrees of freedom, where $J$ equals the number of $\Delta_{i} \mathrm{~s}$ :

$$
\underset{i=1}{J} \chi^{2} \quad \Delta_{i}^{i}=\chi^{2}(J-1)
$$

If the lowest value of $x^{2}(J-1)$ that can be obtained in this fashion is statistically significant, then the categorical perception model does not adequately describe performance in the 2-AIT. In other words, a significant minimal $\chi^{2}(\mathrm{~J}-1)$ would mean that a good fit to the data cannot be obtained by assuming that the various $\Delta_{\mathrm{i}} \mathrm{s}$ were treated as indistinguishable. 1

The outcome of this test for each $\mathbf{S}$ is presented in Table 2. The analysis yielded one categorical and six noncategorical perceivers. The extremely low $\theta$ giving the best fit for the categorical perceiver indicates that she placed nearly all of her $\Delta_{i} s$ in the $G$ category. It is impossible to tell whether such performance reflects her best effort at the task. Given the abundance of noncategorical perceivers found, it is tempting to suppose that the isolated case reflects a disinterested or poorly motivated $S$.

Mentioned above was the assumption that no $S$ would hear fewer $/ \mathrm{b} / \mathrm{s}$ or $/ \mathrm{g} / \mathrm{s}$ on the 2-AIT than on the 3-AIT. When this assumption is violated, indicating that the $\mathrm{S}$ narrowed either her /b/ or /g/ category, or both, the $\theta$ range of 0.00 to 1.00 might exclude that constant which would permit an acceptable fit are presented in Fig. 2.
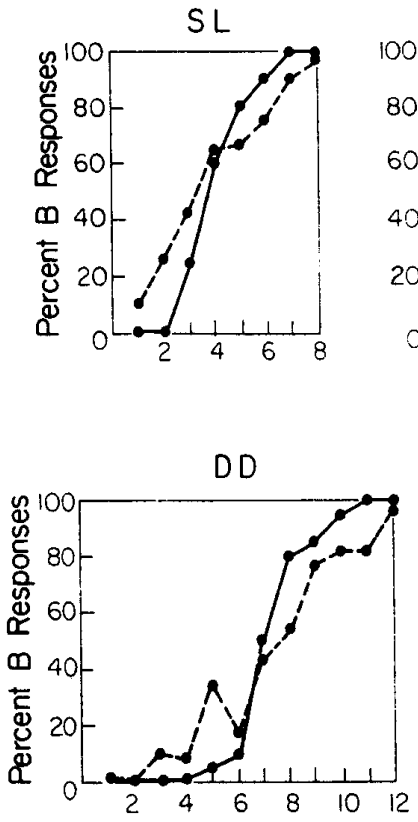
the same stimulus across Ss.
Thus, for only one of seven Ss was it possible to provide a satisfactory fit to the obtained data while maintaining the assumption that all $/ \mathrm{d} / \mathrm{s}$ were perceived alike. It remains to be demonstrated that the perceived distinctions among the $/ \mathrm{d} / \mathrm{s}$ were systematic, which would imply that Ss could appropriately locate allophonic $/ \mathrm{d} / \mathrm{s}$ along their continuum of variation. This can be shown by solving for $\theta$ in Eqs. 1 and 2 for each $\Delta_{i}$ for each $S$. The results, presented in Table 3, indicate that for the six noncategorical perceivers $\theta$ tended to increase regularly toward the $/ \mathrm{b} /$ end of the continuum, while $\theta$ hovered near zero for the categorical perceiver.

The findings may be summarized as follows: A comparison of oddity discrimination and 3-AIT data produced a sufficient number of "categorical perceivers" to confirm the suitability of apparatus and materials for the tasks at hand. If perception was, in fact, categorical, then whenever a $S$ in the 2-AIT heard some representation of $/ d /$, his tendency to label it B (or, reciprocally, G) should be constant across all such stimuli. Identification functions predicted on this basis failed to fit obtained functions for six of seven Ss. Furthermore, the tendency to label an allophonic $/ \mathrm{d} /$ as $B$ was found to increase regularly toward the $/ \mathrm{b} /$ end of the continuum for these six Ss,
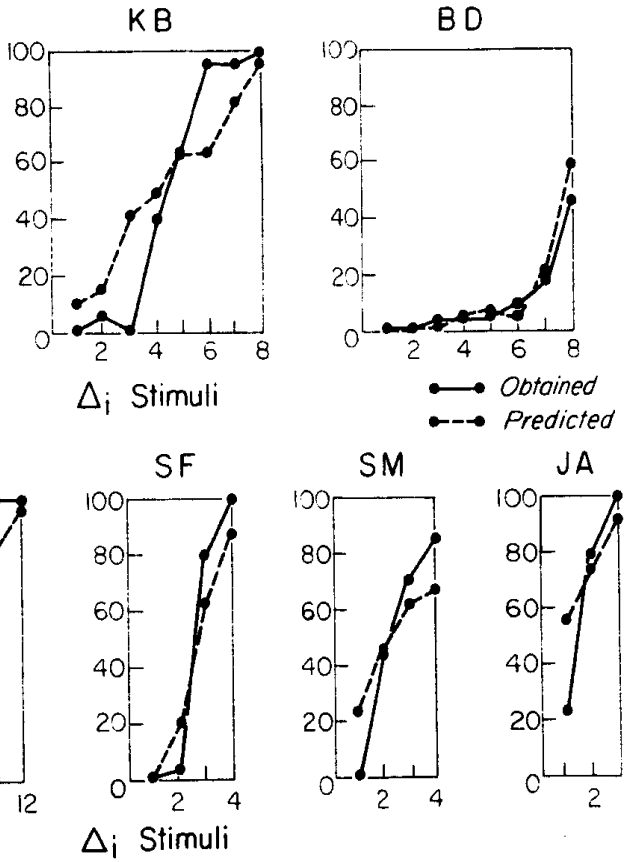

Fig. 2. Predicted and obtained B responses (G response functions are simply the inverses of these). The $/ \mathrm{b} /$ end of the continuum lies to the right of each presented portion. Note: A given stimulus number does not necessarily denote 
Table 3

Obtained $\theta$ Values for $\Delta_{i}$ Stimuli in the 2-AIT

$\Delta_{\mathbf{i}}$ Stimulus Number*

\begin{tabular}{|c|c|c|c|c|c|c|c|c|c|c|c|c|}
\hline $\mathbf{S}$ & $\mathbf{1}$ & 2 & 3 & 4 & 5 & 6 & 7 & 8 & 9 & 10 & 11 & 12 \\
\hline SM & .00 & .40 & .55 & .73 & & & & & & & & \\
\hline JA & -1.67 & 1.00 & 2.00 & & & & & & & & & \\
\hline BD & .00 & .00 & .00 & .06 & .05 & .00 & .06 & .28 & & & & \\
\hline SF & -.20 & -.56 & .33 & 1.00 & & & & & & & & \\
\hline DD & .00 & .00 & .00 & .00 & .00 & .22 & .47 & .77 & .67 & .83 & 1.25 & 1.00 \\
\hline SL & .00 & .00 & .38 & .60 & .79 & .86 & 1.00 & 1.00 & & & & \\
\hline $\mathbf{K B}$ & .00 & .20 & .00 & .46 & .60 & .94 & .89 & 1.00 & & & & \\
\hline
\end{tabular}

*Stimulus numbers do not necessarily denote the same stimuli across Ss.

suggesting that they could, in effect, perceive the location of the various /d/s along the F2 transition continuum. Although the present study demonstrated noncategorical perception of only one voiced stop, there is no reason to suspect that $/ d /$ is unique among voiced stops in this respect.

While the above results rejected the categorical perception hypothesis, they do not necessarily indicate that the voiced stops are (or can be) perceived continuously. To conclusively demonstrate continuous perception of the voiced stops, one must show that the accuracy of simultaneous discrimination remains constant across the stimulus range for any given acoustic interval separating the sounds. The present study did not directly measure simultaneous discrimination, vary the size of the acoustic interval, or closely examine the entire stimulus range.

Nonetheless, because the continuous perception hypothesis remains an interesting possibility, it will be briefly sketched here. When the present Ss had to remember previously presented stimuli for comparisons in the oddity task, most appeared to remember phonemes rather than specific allophones. Perhaps this phenomenon is better described as "categorical remembering" than as categorical perception. That is, the stimuli might well be perceived continuously (indicating a stage in speech processing in which allophone-specific information in the signal is briefly available to conscious inspection) and then stored categorically as phonemes. This line of reasoning suggests the following conception, which is consistent with the results of research bearing on Liberman et al's (1967) motor theory of speech perception. Phoneme-specifying information in the acoustic signal normally arrives at a tremendous rate and must be rapidly encoded if it is to be remembered long enough for the message to be intelligible. If the memory code is, in part, an articulatory code, some of the information which does not fit this code will be quickly lost by the listener. It appears that information specifying a particular acoustic version of a consonant may be lost in this way, while the consonant itself is retained in the articulatory code. Thus, to the degree that articulation of a given phoneme is categorical, acoustic representations of that phoneme could be stored categorically, although perceived continuously.

\section{REFERENCES}

BARCLAY, J. R. Noncategorical perception of a voiced stop consonant. Proceedings of the 78th Annual Convention of the American Psychological Association, 1970, 9-10.

FRY, D. B., ABRAMSON, A. S., EIMAS, P. D., \& LIBERMAN, A. M. The identification and discrimination of synthetic vowels. Language \& Speech, $1962,5,171.189$.

HALWES, T. G. Effects of dichotic fusion on the perception of speech. Supplement to: Status report on speech research, Haskins Laboratories, September 1969. LIBERMAN, A. M., COOPER, F. S., S H A N K W E I L E R, D . P..ं \& STUDDERT-KENNEDY, M. Perception
of the speech code. Psychological Review, of the speech code.
$1967,74,431-461$.

LIBERMAN, A. M., DELATTRE, P. C., COOPER, F. S., \& GERSTM AN, L. J. The role of consonant-vowel transitions in the perception of the stop and nasal consonants. Psychological Monographs, 1954, 68(8, Whole No. 379).

LIBERMAN, A. M." HARRIS, K. S., HOFFMAN, H. S., \& GRIFFITH, B. C. The discrimination of speech sounds within and across phoneme boundaries. Journal of Experimental Psychology, $597,54,358-368$.

LIBERMAN, A. M.. HARRIS, K. S KINNEY, J. A., \& LANE, $H$, The discrimination of relative on set time of the components of certain speech and nonspeech patterns. Journal of Experimental Psychology, 1961, 61, 379-388.

STEVENS, K. N., OHMAN, S. E. G., \& LIBERMAN, A. M. Identification and discrimination of rounded and unrounded vowels. Journal of the Acoustical Society of America, 1963, 35, 1900. (Abstract)

STRANGE, W., \& HALWES, T. Confidence ratings in speech perception research: Evaluation of an efficient technique for discrimination testing. Perception \& Psychophysics, 1971, 9, 182-186.

STUDDERT-KENNEDY, M., LIBERMAN, A. M., HARRIS, K. S., \& COOPER, F. S Motor theory of speech perception: A reply to Lane's critical review. Psychological Review, 1970, 77, 234-249.

\section{NOTE}

1. This statistical test was suggested by John I. Yellott, Jr.

Received for publication September 15 1971.) 